\title{
Proteogenomics analysis reveals specific genomic orientations of distal regulatory regions composed by non-canonical histone variants
}

\author{
Kyoung-Jae Won ${ }^{1,2+}$, Inchan Choi ${ }^{1,2,5 \dagger}$, Gary LeRoy ${ }^{3+}$, Barry M Zee ${ }^{3,4}$, Simone Sidoli ${ }^{4}$, Michelle Gonzales-Cope ${ }^{3,4}$ \\ and Benjamin A Garcia ${ }^{4 *}$
}

\begin{abstract}
Background: Histone variants play further important roles in DNA packaging and controlling gene expression. However, our understanding about their composition and their functions is limited.

Results: Integrating proteomic and genomic approaches, we performed a comprehensive analysis of the epigenetic landscapes containing the four histone variants H3.1, H3.3, H2A.Z, and macroH2A. These histones were FLAG-tagged in HeLa cells and purified using chromatin immunoprecipitation (ChIP). By adopting ChIP followed by mass spectrometry (ChIP-MS), we quantified histone post-translational modifications (PTMs) and histone variant nucleosomal ratios in highly purified mononucleosomes. Subsequent ChIP followed by next-generation sequencing (ChIP-seq) was used to map the genome-wide localization of the analyzed histone variants and define their chromatin domains. Finally, we included in our study large datasets contained in the ENCODE database. We newly identified a group of regulatory regions enriched in H3.1 and the histone variant associated with repressive marks macroH2A. Systematic analysis identified both symmetric and asymmetric patterns of histone variant occupancies at intergenic regulatory regions. Strikingly, these directional patterns were associated with RNA polymerase II (PollI). These asymmetric patterns correlated with the enhancer activities measured using global run-on sequencing (GRO-seq) data.
\end{abstract}

Conclusions: Our studies show that H2A.Z and H3.3 delineate the orientation of transcription at enhancers as observed at promoters. We also showed that enhancers with skewed histone variant patterns well facilitate enhancer activity. Collectively, our study indicates that histone variants are deposited at regulatory regions to assist gene regulation.

\section{Background}

The eukaryotic genome is packaged in the nucleus as chromatin, a dynamic arrangement which serves to compact the DNA. Chromatin structure is highly complex as, while packaged, is accessible for selective gene expression and DNA repair. Moreover, chromatin is highly dynamic during chromosome condensation processes such as mitosis and meiosis [1]. The fundamental unit of chromatin is the nucleosome. Nucleosomes are composed of an octamer of histone proteins comprised of two copies each of H2A, H2B, H3, and H4 [2]. Histone Nterminal tails are exposed outside the nucleosomes, and

\footnotetext{
*Correspondence: bgarci@mail.med.upenn.edu

${ }^{\dagger}$ Equal contributors

${ }^{4}$ Epigenetics Program, Department of Biochemistry and Biophysics, Perelman School of Medicine, University of Pennsylvania, Philadelphia, PA 19104, USA Full list of author information is available at the end of the article
}

they are heavily modified by dynamic post-translational modifications (PTMs). The deposition of such PTMs modulates chromatin structure, which directly affects the abovementioned DNA-related events [3,4]. Histone PTMs are also among the major drivers of epigenetic memory, as they can be inherited after cell division [5]. Aberrations in PTM relative abundance have been found in several diseases [6,7], which highlights the direct link between histone marks and cell phenotype.

In addition to the canonical histones, there are also protein variants encoded by separate genes [8]. These variants play further important roles in DNA packaging and controlling gene expression [9]. For instance, histone $\mathrm{H} 2 \mathrm{~A} . \mathrm{Z}$ replaces canonical $\mathrm{H} 2 \mathrm{~A}$ at some $5^{\prime}$ end of both active and inactive genes [10-15]. Recent studies also identified that H2A.Z is enriched at active enhancers, destabilizing the local nucleosome structure and facilitating 
nucleosome removal $[16,17]$. Histone H3.3 is specially enriched at transcriptionally active genes as well as regulatory elements [18-22]. Also, unstable H2A.Z/H3.3 doublevariant-containing nucleosomes were reported at active promoters, enhancers, and insulator regions [23]. Another $\mathrm{H} 2 \mathrm{~A}$ variant, macroH2A, is enriched for the inactive $\mathrm{X}$ chromosome, and therefore, it has been mainly associated with heterochromatic regions [24,25]. More recently, macroH2A has also been shown to activate genes, although it was still closely associated with the silencing mark H3K27me3 [26]. Such complex panorama of histone PTMs and variants calls for further studies to more accurately define the combinatorial preferences of histone variants and their function for gene regulation.

In order to understand the strategic deposition of histone proteoforms and their functional roles, we quantitatively investigated using chromatin immunoprecipitation coupled to mass spectrometry (ChIP-MS) the composition at single-nucleosome resolution of histone-variantcontaining mononucleosomes. Moreover, such mononucleosomes were genome-wide mapped using chromatin immunoprecipitation followed by next-generation sequencing (ChIP-seq). In these experiments, we employed HeLa cells expressing either FLAG-tagged canonical histone H3, H3.3, canonical histone H2A, and H2A.Z. Moreover, FLAG-affinity-purified mononucleosomes were analyzed by ChIP-MS (Figure 1A, B) to quantitatively determine histone PTM composition [27]. Hence, our proteogenomic approach allowed us to define chromatin domains containing combinations of histone variants. In particular, we mapped and determined the composition of domains enriched in histone H3.1 and macroH2A, which last is known as repressive signature. More importantly, we observed directional profiles of histone variants, where histone H2A.Z occurred ahead of H3.3 in gene enhancers. This directional pattern cooccurred with the enrichment of RNA polymerase II (PolII), suggesting that PolII has orientation at enhancers and histone variants reflect its transcriptional direction.

\section{Results and discussion \\ Determination of the relative abundance of histone variants}

Nucleosomes contain two copies of each core histone type (that is, H4, H3, H2A, and H2B). We used quantitative MS to analyze the ratio of the different $\mathrm{H} 3$ and $\mathrm{H} 2 \mathrm{~A}$ variants in the FLAG-purified mononucleosomes. As expected, canonical $\mathrm{H} 2 \mathrm{~A}$ was found to be the most abundant variant in nucleosomes purified with either FLAG-H2A or FLAG-H3.1 (Figure $1 \mathrm{C}$ and Additional file 1: Table S1). H2A.Z was not observed to be enriched in mononucleosomes purified with histone H3.3, even though previous studies indicate that it should be enriched at enhancers of expressed genes [16,17]. We found a portion of H2A.Z co-existing with H3.3, but this
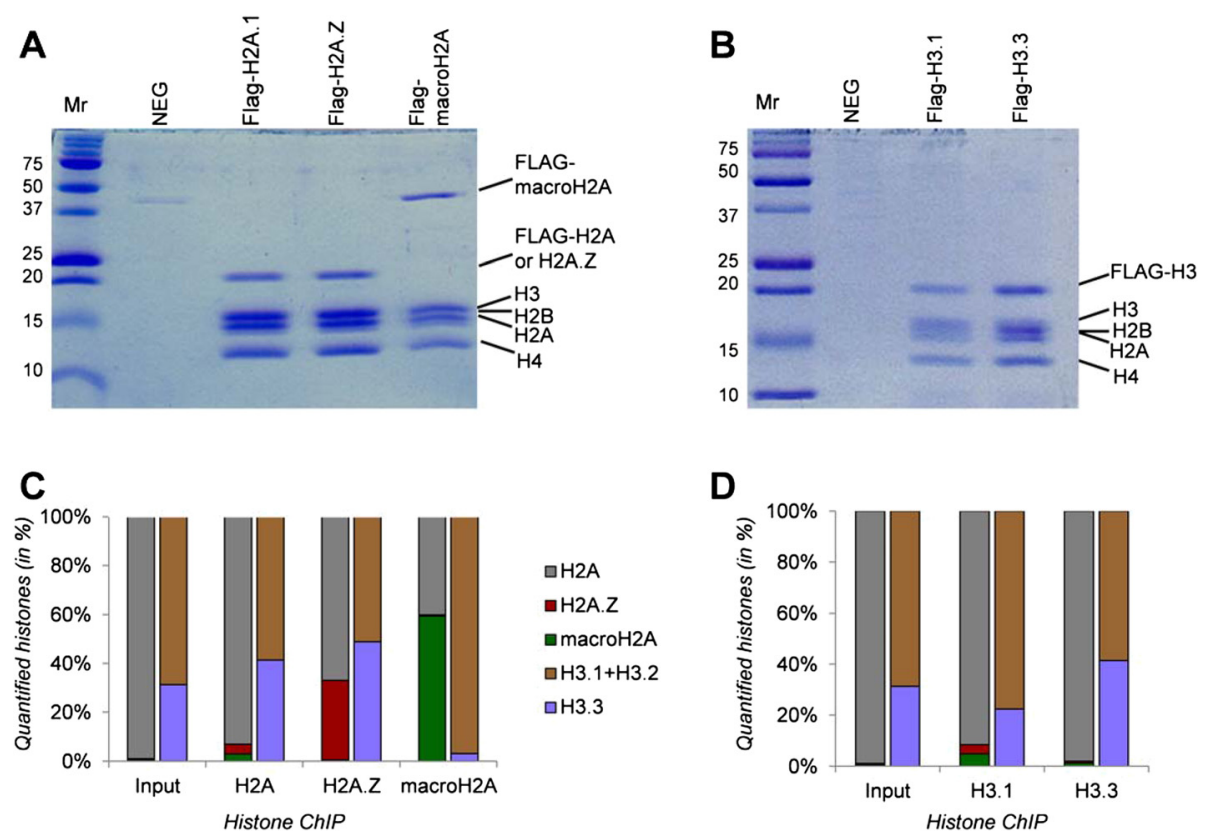

Figure 1 Analysis of immunoprecipitated mononucleosomes. Coomassie staining gel representing protein composition of (A) FLAG-H2Aand (B) FLAG-H3-immunoprecipitated histone samples. NEG represents negative control. (C) Relative abundance of canonical histone H2A (gray), H2A.Z (red), macroH2A (green), canonical histone H3 (H3.1 + H3.2, brown), and H3.3 (violet) calculated from the ChIP-MS analysis of H2A FLAGtagged histones and (D) H3 FLAG-tagged histones. 
portion is very small and likely quite specialized. Canonical $\mathrm{H} 2 \mathrm{~A}$ makes up about $50 \%$ of the $\mathrm{H} 2 \mathrm{~A}$ population in mononucleosomes purified with FLAG-H2A.Z or FLAG-macroH2A, suggesting that nucleosomes that contain these variants are asymmetric, containing one copy of canonical H2A (Figure 1C and Additional file 1: Table S1). Surprisingly, a small fraction of mononucleosomes were detected to possess both H2A.Z and macroH2A. On the other hand, analysis of H3.3-containing nucleosomes shows that canonical histone $\mathrm{H} 2 \mathrm{~A}$ is the most abundant form, followed by H2A.Z, and then finally macroH2A (Figure 1C and Additional file 1: Table S1).

We also quantified the relative ratios of H3.1 and H3.2 versus H3.3 in the FLAG-purified nucleosomes by using MS (Figure 1D). To do so, we utilized a peptide that was common in the H3.1 and H3.2 variants (a.a. 27 to 40) yet differed from the H3.3 variant by one amino acid (see the 'Methods' section). Therefore, we were not able to distinguish H3.1 from H3.2. From our calculations, H3.1 FLAG-purified nucleosomes contained histone H3 variants with a ratio of roughly 1:3 (H3.3:canonical H3) (Figure 1D and Additional file 1: Table S1). This suggests that most of the nucleosomes containing the variant $\mathrm{H} 3.3$ are asymmetric, containing one copy of H3.1 or H3.2 partnered with one copy of H3.3. This conclusion was further supported by the fact that approximately half of the FLAG H3.3-purified nucleosomes contained either H3.1 or H3.2 (Figure 1D and Additional file 1: Table S1). The most significant observation regarding the $\mathrm{H} 3$ variants was found in the FLAG macroH2Apurified nucleosomes; these nucleosomes contained only approximately $3 \% \mathrm{H} 3.3$, suggesting that $\mathrm{H} 3.3$ is rarely found in nucleosomes containing the repressive macroH2A variant (Figure 1D and Additional file 1: Table S1). This result was further supported by the fact that Flag H3.3-purified nucleosomes contained very low levels of macroH2A (approximately $1 \%$ ).

\section{Determination of histone PTM relative abundance}

By using ChIP-MS results (Figure 2 and Additional file 2: Table S2), we investigated the relative abundance of histone PTMs in H2A.Z- and H3.3-containing mononucleosomes. Briefly, we observed an enrichment of active marks in such nucleosomes as compared to the global chromatin levels and, in particular, to nucleosomes containing the histone variant macroH2A. For instance, the activating mark H3K4me2 mark was highly enriched in nucleosome ChIPed with H3.3 or H2A.Z (11.5-fold in H3.3 and 19.8fold in H2A.Z as compared with the genomic chromatin levels). This data was interesting considering that H2A.Z was not enriched in mononucleosomes purified with the $\mathrm{H} 3$ variant $\mathrm{H} 3.3$, indicating that $\mathrm{H} 3.3$ and $\mathrm{H} 2 \mathrm{~A} . \mathrm{Z}$ may occupy distinct chromatin regions marked by H3K4me2. H3K4me3 was found to be enriched almost 30-fold in
H2A.Z-purified nucleosomes as compared to global input (Figure 2A and Additional file 2: Table S2), which was consistent with previous observations that investigated active promoters [11]. A similar trend was observed for H3K36me3 in H3.3- and H2A.Z-purified nucleosomes, which was about $7 \%$ and $15 \%$ of the total histone $\mathrm{H} 3$, respectively. In genomic chromatin and macroH2Acontaining nucleosomes, H3K36me3 was only $4 \%$ and $1 \%$, respectively (Figure 2 and Additional file 2: Table S2). This was not surprising, as H3K36me3 is enriched downstream to the transcriptional start sites (TSSs) of active genes, which are the same genomic regions where H2A.Z is enriched [11]. Interestingly, H3.3-purified nucleosomes were less enriched in H3K36me3 than nucleosomes purified with H2A.Z, even though both H3.3 and H3K36me3 generally mark actively transcribed regions [11,18-21].

As compared to the total chromatin, both H3.3- and H2A.Z-purified nucleosomes were enriched for the activating marks $\mathrm{H} 3 \mathrm{~K} 9 \mathrm{ac}$ and $\mathrm{H} 3 \mathrm{~K} 27 \mathrm{ac}$ (4.3- and 2.4-fold changes for H3K9ac and 1.8- and 2.4-fold changes for H3K27ac, respectively) (Figure 2 and Additional file 2: Table S2). We also observed a dramatic enrichment of $\mathrm{H} 4 \mathrm{~K} 16 \mathrm{ac}$ in nucleosomes purified with H3.3 (approximately 50\%) and H2A.Z (approximately 40\%) as compared to this modification in genomic chromatin (approximately 20\%). This confirmed once again what we expected, as H3.3, H2A.Z, and H4K16ac are all enriched in gene bodies [28]. Moreover, H4K16ac is highly enriched in nucleosomes bound by the BET family bromodomain containing proteins (Brd2, Brd3, and Brd4), which are bound to and assist gene transcription by PolII [29]. The enrichment of H4K16ac was present in tandem with combinations of H4K5ac, H4K8ac, and H4K12ac in H3.3- and H2A.Z-purified nucleosomes (Figure $2 \mathrm{~B}$ and Additional file 2: Table S2). Finally, the repressive mark H3K27me3 was enriched approximately threefold changes in macroH2A-purified nucleosomes as compared to genomic chromatin (approximately $18 \%$ in macroH2A-purified $v s$. approximately $6 \%$ in genomic or approximately $5 \%$ in H2A.1-purified nucleosomes). Moreover, we observed an inverse relationship between H3K4me2/3 and H3K27me3 on nucleosomes purified with either H2A.Z or macroH2A. Conversely, H3K27ac levels in macroH2A ChIPs were depleted (0.26\%) as compared to the H3K27ac levels found in canonical H2A.Z ChIPs. Taken together, our data demonstrate that the trends of the major PTMs we observed in histones $\mathrm{H} 3$ and $\mathrm{H} 4$ were similar for H2A.Z and H3.3.

\section{Histone variant genome-wide profiles}

Our proteomic analyses further questioned how the cooccupying histone variants were represented in the genome. For this, we used deep sequencing approaches to map the FLAG-tagged histone variants in the genome. 


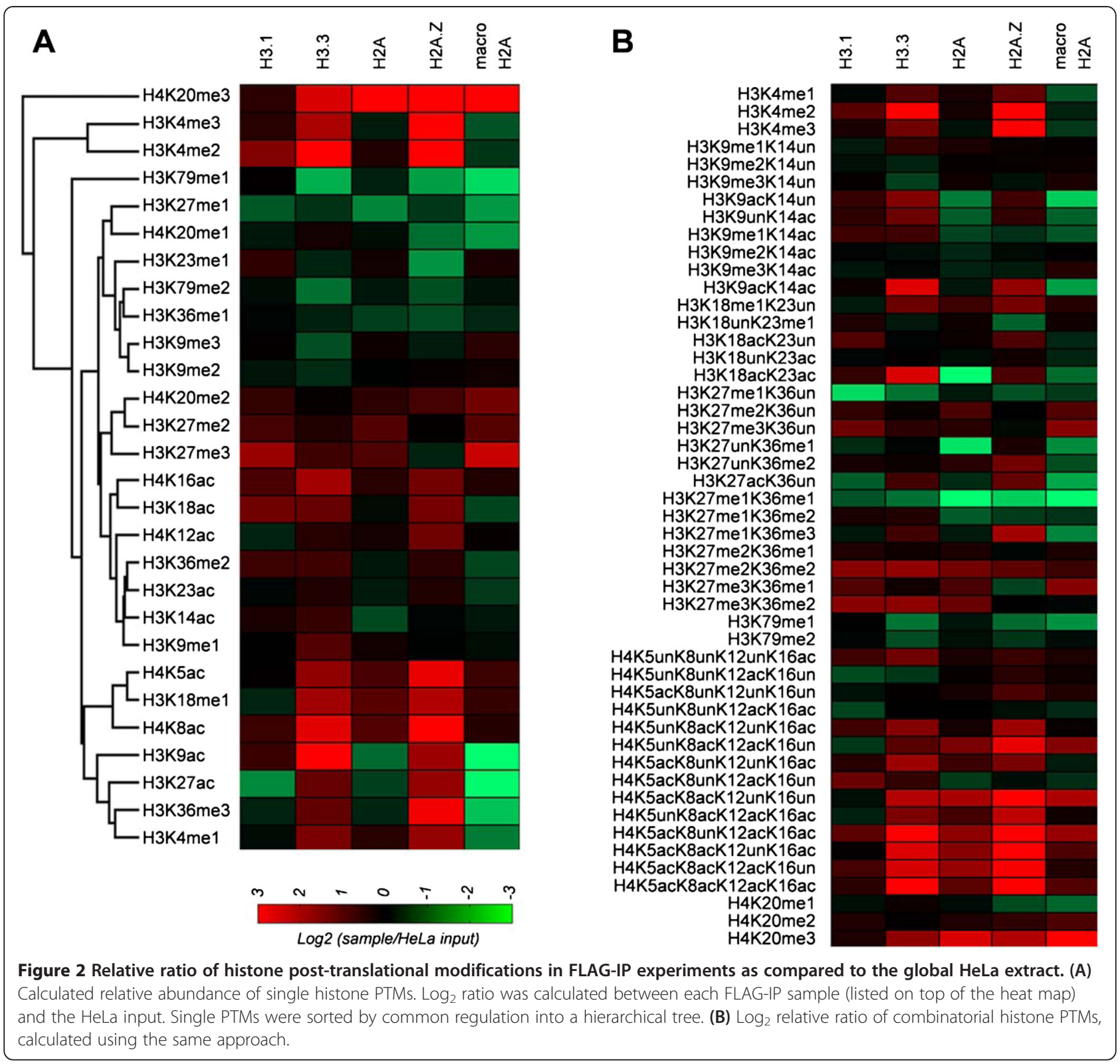

First, we asked how the histone variants are positioned around genes. After sorting the annotated Refseq genes based on their expression levels, we investigated the histone variant levels around the genes. H2A.Z was highly enriched around the annotated TSSs of active genes but absent in inactive genes (Figure 3A and Additional file 3: Figure S1). This was consistent with the previous genome-wide surveys $[11,23]$. The sharp enrichment of H2A.Z marked the two nucleosomes flanking the nucleosome-free regions (NFRs) at active promoters $[11,30]$. In the gene body, H2A.Z was absent regardless of their expression levels (Additional file 3: Figure S1).

Both H3.1 and H3.3 were depleted around the center of TSSs of active genes (Figure 3A and Additional file 3:
Figure S1). These results differ from the previous study in mouse embryonic stem cells (mESCs) where H3.3 showed strong bimodal enrichment at the TSSs of active genes [16], but they are in agreement to the previous genome-wide study in HeLa cells [23]. At transcription termination sites (TTSs), we also found a depletion of H3.1 and H3.3 [31]. In general, H3.1 and H3.3 levels correlated with gene expression levels in the gene body. H3.3 levels were increased towards the 3' end of the genes, consistent with previous studies [21,23]. While H2A.Z was sharply enriched around TSSs, the enrichments of H3.1 and H3.3 were modest. Also, we confirmed a negative correlation of the macroH2A with gene expression levels [32]. 

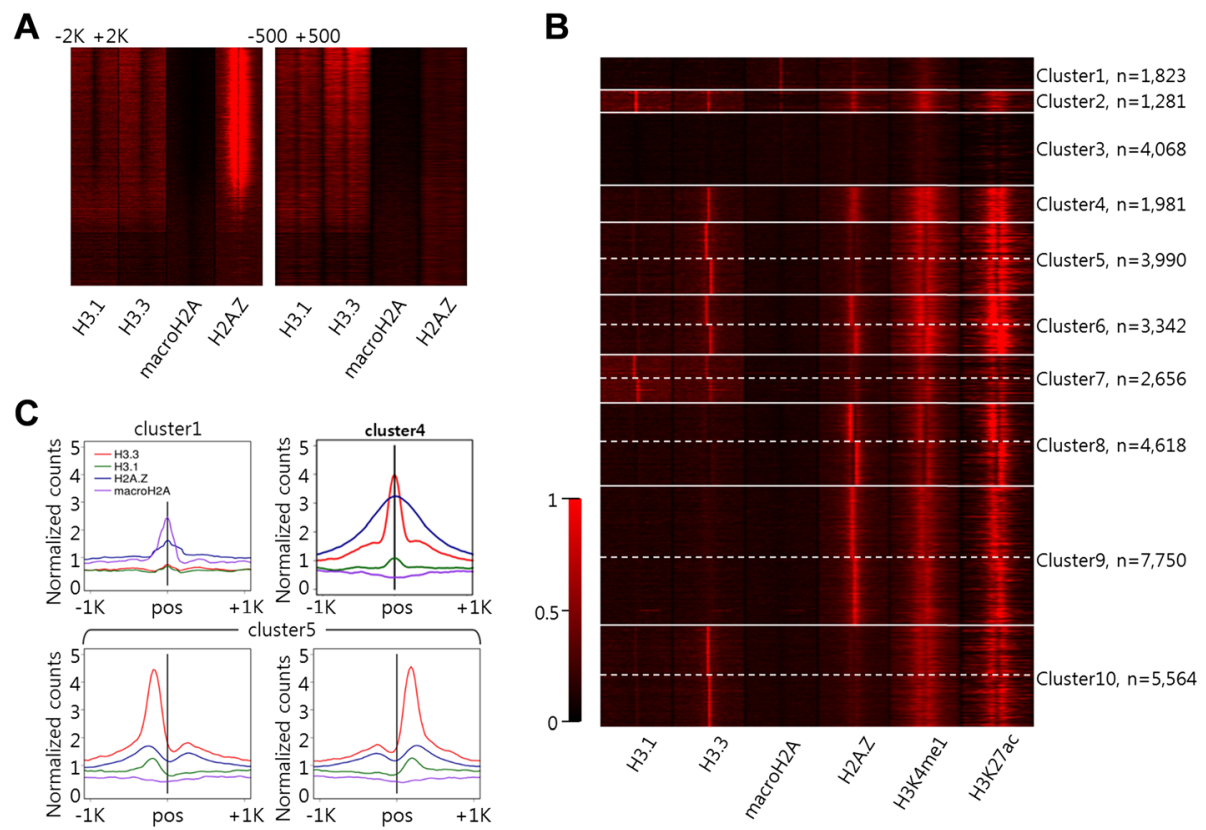

Figure 3 Genomic profiles of histone variants. (A) Distribution of ChIP-seq reads at annotated TSSs $( \pm 2 \mathrm{~K})$ and TTSs $( \pm 500)$ and (B) at distal regulatory regions. We clustered DHSs located in the intergenic region. We identified 16 groups and rearranged them to 10 clusters based on their profiles. Various compositions of histone variants were found. Clusters 5 to 10 are composed of 2 mirroring groups. After clustering based on histone variants, we aligned histone modification. Histone variants are off-centered for the mirroring clusters (clusters 5 to 10), suggesting orientation at regulatory regions. (C) Symmetric and asymmetric profiles of histone variants. Clusters 1 and 4 show symmetric profiles with various compositions of histone variants. Cluster 5 shows mirroring asymmetric profile. All profiles for all clusters are shown in Additional file 3: Figure S2.

We then investigated if histone variants are enriched at promoter distal ( $>2 \mathrm{kbp}$ from known TSSs) regulatory regions. For this, we retrieved the map of DNaseI hypersensitive sites (DHSs) in HeLa cells from ENCODE [33]. DHSs potentially demarcate regulatory elements, including promoters, enhancers, silencers, insulators, and locus control regions [34]. We identified a total of 94,600 DHSs using Homer [35]. Among them, 37,073 DHSs were located distal $(>2 \mathrm{kbp})$ to the known TSSs, TTSs, and outside the body of the annotated mRNA and the long non-coding RNAs (lncRNAs). Monitoring the four histone variants (H2A.Z, macroH2A, H3.1, and H3.3) at the distal regulatory regions, we defined 16 groups (Figure 3B). We then further characterized such clusters by examining the histone PTMs H3K4me1 and H3K27ac, markers for enhancers [36,37]. Based on the proteomic study (Figure 2), we expected H2A.Z and H3.3 are with activating histone modification marks. Our results showed various combinations of histone variants at these distal regulatory regions. As expected, majority of the distal DHSs were enriched for H3.3 and/or H2A.Z as well as H3K27ac indicating that these histone variants are important for enhancer function. Also, we found clusters marked by H3.1 (clusters 2 and 7) or even with repressive macroH2A mark (cluster 1). Contrary to our proteomic results (Figure 2B), clusters 2 and 7 are enriched for H3.1 and activating H3K27ac mark at a certain level. The clustering results indicate diverse epigenetic codes composed of histone variants at distal regulatory regions.

Histone variants have symmetric and asymmetric patterns at distal regulatory regions

Histone H3.3 and other histone variants were observed to have asymmetric profiles (Figure 3B, C and Additional file 3: Figure S2). The clusters 5 to 7 showed that H3.3 and H2A.Z were skewed to one side. We also checked the average profiles of p300, H3K27ac, and DHSs at each cluster. The H3K27ac profiles were enriched on the side where the H3.3 peak was located (Additional file 3: Figure S3) even though the skewness was less dramatic as compared to histone variant profiles. The DNaseI and histone acetyltransferase p300 profiles, marker for enhancers $[37,38]$, were centered at the DHSs regardless of the pattern of the histone variants (Additional file 3 : Figure S3), confirming that transcriptional co-factors are not biased to a single direction. Other transcription factors analyzed did not show asymmetric patterns either (data not shown), further indicating that the skewness in the histone variant profiles was independent from transcription factors and their co-factors.

To further investigate the association of histone variants with gene regulation, we examined PolII occupancy, which was found to be skewed towards the peak of H3.3 
and H2A.Z (clusters 5 to 10) (Figure 4A and Additional file 3: Figure S4). In clusters 1 to 4, PolII peaked at the center of DHSs. In these clusters, H3.3 either peaked directly over the DHSs (clusters 2 and 4) or showed no enrichment over the entire $2 \mathrm{~Kb}$ region surrounding the DHSs (clusters 1 and 3). In clusters 5, 6, 7, and 10, PolII was skewed towards the peak of H3.3. This suggests that the position of $\mathrm{H} 3.3$ is related to transcriptional orientation at distal regulatory regions. Figure $4 \mathrm{~B}$ shows an example of skewness of $\mathrm{H} 3.3$ in association with PolII orientation. H3.3 is located on a side of the DHS, and PolII peak was observed between the DHS and H3.3 peak, as shown in the profile. We validated this observation by performing ChIP-qPCR of the five regions around the DHSs. The qPCR experiment confirmed that PolII and H3.3 were not symmetric at a potential enhancer and skewed towards the same direction (Figure 4C).

\section{Role of chromatin domains containing studied histone variants}

We investigated the enhancer activities of these potential regulatory using the global run-on sequencing (GROseq) data in HeLa cells [39]. GRO-seq can measure the transcription levels at enhancers (or eRNA [40,41]). All clusters showed bidirectional transcripts in their eRNA profiles. Especially, we observed modest bias of the strand-specific transcripts that matched with the PolII orientation in the asymmetric clusters (Figure 4A and Additional file 3: Figures S4 and S5). The enhancer activity and the PolII levels of the cluster with macroH2A (cluster 1 ) were very weak. Actually, the eRNA levels of cluster 1 were even smaller than cluster $3(P$ value $=$ $3.4 \mathrm{e}-37)$, where we did not find any strong signal of histone variants. This further confirmed the repressive role of macroH2A at distal regulatory regions. Both clusters 5 and 10 were enriched for H3.3, but eRNA levels were significantly larger in cluster 5 where the skewness was more pronounced $(P$ value $=1.3 \mathrm{e}-13)$. We also observed stronger eRNA levels for cluster 8 than cluster 9 , where skewness was more strongly observed $(P$ value $=$ $6.4 \mathrm{e}-11)$. Collectively, these suggest that skewed histone variants facilitate enhancer activity.

DHSs represent open chromatin regions in the genome. Unexpectedly, clusters 2 and 4 had histone variants enriched at DHSs, which are potential nucleosome-free regions. Interestingly, the dip in the bimodal pattern for H3K27ac was rather shallow or lost for clusters 2 and 4 (Additional file 3: Figure S3). Previous studies have reported that nucleosome core particles (NCPs) containing both H3.3 and H2A.Z are unstable and that such NCPs were enriched at nucleosome-depleted regions [23,42]. Thus, clusters 2 and 4 may represent an example of the unstable histone variants at DHSs.

Finally, we investigated the occupancy of transcription factors into the clusters we identified. We calculated the hyper-geometric $P$ value using the peak information for each cluster. Interestingly, we observed that transcription factors prefer specific epigenetic environments (Additional file 3: Figure S6); clusters 1, 2, 3, and 7 were uniquely populated with specific factors such as CTCF and Zzz3. CTCF is involved in many functions including transcriptional activation/repression, insulation, imprinting, and forming higher-order structures [43]. CTCF binding was significantly observed at cluster 3 , where the levels of the activating histone marks H3K4me1 and H3K27ac were depleted (Additional file 3: Figure S5),
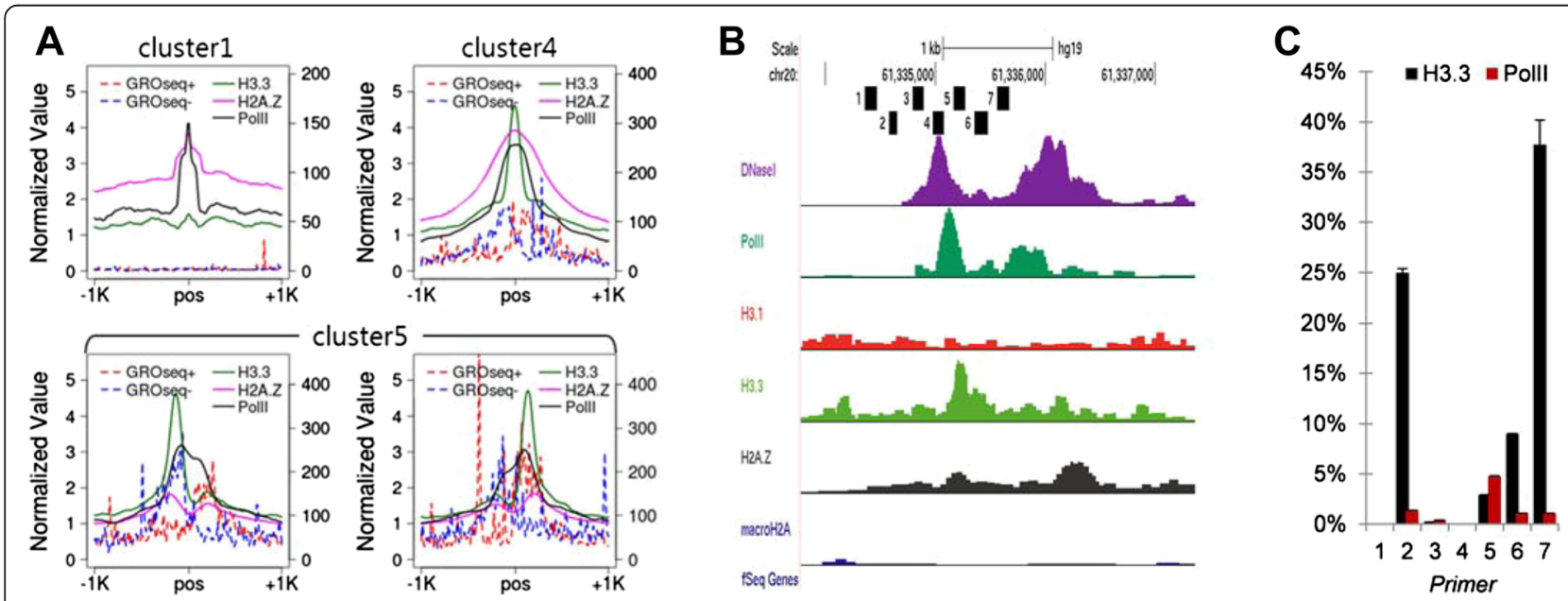

Figure 4 Histone variants are associated with Polll orientation. (A) The profiles of H3.3 and H2A.Z are associated with the pattern of Polll and enhancer transcripts. For symmetric clusters, Polll is located at the center. For asymmetric clusters, Polll is skewed to the direction of the peaks of histone variants. Transcripts at enhancers show bidirectional patterns. Strand-specific transcripts are stronger in the asymmetric clusters. (B) The screenshot of histone variant around DNasel. H3.3 is enriched more to the right side of the peak of DNasel. Polll has its peak to the right side of DNasel. (C) ChIP-qPCR validation on the seven regions. Polll enrichment is skewed towards the right side. 
presumably associated with the insulator function. Interestingly, cluster 7 was uniquely enriched for CTCF, suggesting that the enrichment of $\mathrm{H} 3.1$ is designated to a specific function of CTCF. We also found Zzz3 significantly enriched in clusters 1 and 2 as compared to the other clusters, which are potentially containing unstable nucleosome at DHSs (Additional file 3: Figure S6). Overall, this suggests that Zzz3 may associate with other factors when forming closed chromatin structure.

\section{Conclusions}

Histone non-canonical variants play a major regulatory role in mammalian genomes. For example, H2A.Z is enriched at active promoters as well as active enhancers marked by H3K27ac, and H3.3 was found enriched at the peaks of H2A.Z [16]. In this paper, we dissected the composition of histone variants using both proteomics and genomics strategies. Our quantitative MS results revealed that activating histone PTMs were highly enriched on H2A.Z-containing and H3.3-containing mononucleosomes, while such marks were generally depleted in macroH2A-containing mononucleosomes. Conversely, macroH2A-containing mononucleosomes were enriched for repressive histone PTMs.

We further investigated the distribution of histone variants in the genome especially at distal regulatory regions. We identified diverse compositions of four histone variants at potential regulatory regions. Besides H3.3 and H2A.Z, which have been known to be enriched at regulatory regions $[16,21]$, we additionally found regulatory regions enriched in $\mathrm{H} 3.1$ or macroH2A. The regulatory regions enriched for macroH2A were depleted for active histone marks and enhancer activities, suggesting a repressive role for this element (cluster 1, Figure 3C). H3.1 was observed in two clusters: one in the closed chromatin structure (cluster 2) and one with strong enrichment of CTCF. This evidenced that histone H3.1 has still several unknown functions at distal regulatory regions. Unexpectedly, we also found histone variants at the center of DHSs, potential nucleosome-free regions. Specifically, we observed the centers of some DHSs enriched for H2A.Z and H3.3 (clusters 2 and 4). These regulatory elements may be associated with unstable nucleosomes containing H3.3/H2A.Z double variant at DHS [23].

Importantly, we found asymmetric patterns of histone variants. In general, symmetric bimodal patterns have been accepted as profile for activating histone marks $[37,44,45]$. Our results showed a large portion of regulatory regions with asymmetric patterns. These regulatory regions were associated with the skewed patterns of the activating mark H3K27ac. Besides, the asymmetric patterns of histone variants were associated with PolII occupancy. Taken together, this demonstrated that skewed histone variants were not just noise, but such deposition dictates the direction of PolII movement.

Enhancers were originally defined as remote elements that increase transcription independently of their orientation [46,47]. However, some groups already identified a number of enhancer groups with asymmetric histone modification patterns [48]. Other computational models found asymmetric H2A.Z and nucleosome occupancies at CTCF binding sites $[49,50]$. More directly, nascent RNAs at enhancers show both bidirectional and unidirectional transcripts [51]. The $5^{\prime}$ ends of capped RNAs detected by Cap Analysis of Gene Expression (CAGE) in HeLa cells confirmed unidirectional transcripts at enhancers [39]. Nascent RNAs are closely associated with RNA polymerase [52,53]. The asymmetric patterns of eRNA as well as the skewed PolII occupancy in our study suggest that enhancers have directional information. For example, experiments that flipped enhancer sequences changed the activity of promoter in the luciferase assay [54], showing that directional information of the flanking regions around distal regulatory regions is important for gene regulation.

PolII orientation is clearly defined at promoters, where transcriptional orientation defines the asymmetric epigenetic pattern of H3K4me3 towards the direction of transcription [11]. A remarkable observation was made for bidirectional as well as unidirectional promoters in association with histone variants. H2A.Z at active promoters show strong upstream as well as downstream peaks in human and yeast $[11,17,30,55]$, but not in flies [56] or Arabidopsis [57]. The presence of upstream H2A.Z nucleosomes seen in some organisms correlates with bidirectional transcription in yeast and mammals [53,58]. This suggests that histone variants are associated with transcriptional direction. At promoters, moreover, the nucleosome located to the transcriptional direction blocks the movement of PolII [59]. Depletion of H2A.Z from a nucleosome position resulted in a higher barrier to PolII [59]. Enhancers bare similar characteristics with promoters. Besides eRNAs, some enhancers are even with TATA box sites [39]. The nucleosome at distal regulatory region may block the movement of PolII as at promoters. H3.3 as well as H2A.Z may work to remove the barrier for PolII at distal regulatory regions. We additionally investigated the histone variants as well as PolII profiles at active promoters (Additional file 3: Figure S7). The genome-wide profile showed that the PolII and H3.3 profiles at enhancers are strikingly similar to the profile of H2A.Z and PolII at promoters.

In conclusion, why do enhancers have orientation? A DNA looping model has been suggested where promoterenhancer interactions facilitate gene transcription [60-62]. These observations questioned the transcriptional mechanism associated with histone variants and PolII. By 
forming a looping structure, PolII needs to have a preference for its movement as it needs to move towards the transcription orientation of the associated gene (Figure 5). The nucleosome on one side of the regulatory region may block the movement of PolII at distal regulatory regions. H3.3 and H2A.Z may help the movement of PolII by destabilizing the nucleosome that blocks the movement of PolII.

\section{Methods}

\section{Chromatin immunoprecipitation of histone variants}

HeLa S3 cells stably expressing either FLAG-tagged canonical histone H3, H3.3, canonical histone H2A, and H2A.Z were grown in suspension in Joklik media containing 10\% newborn calf serum (Hyclone, GE Healthcare, Little Chalfont, UK), 1\% GlutaMAX (Invitrogen, Carlsbad, CA, USA), and 1\% penicillin-streptomycin, and cells were harvested at log phase. Nuclei were isolated, mononucleosomes were subsequently obtained from these cell lines, and ChIP experiments were performed as described [29]. Briefly, cells were lysed in hypotonic TMSD buffer to isolate nuclei, which were then digested with micrococcal nuclease. The resulting mononucleosomes (Figure 1A, B) were immunoprecipitated with anti-FLAG M2-agarose beads (Sigma-Aldrich, St. Louis, MO, USA) and eluted with FLAG peptide (Sigma-Aldrich, St. Louis, MO, USA).

\section{Preparation of histones for mass spectrometry}

Histone samples, from both ChIP input and ChIP elutions, were prepared as described previously [27], with the exception that the acid extraction step was simply replaced by a boiling step for the ChIP elutions. Briefly, digestion was performed as follows: derivatization reagent was prepared by mixing propionic anhydride with 2-propanol in the ratio $1: 3(v / v)$ and added to the histone sample in the ratio of $1: 2(v / v)$ for $15 \mathrm{~min}$ at $37^{\circ} \mathrm{C}$. This reaction was performed twice to obtain complete labeling. Histones were then digested with trypsin (enzyme:sample ratio of 1:20, overnight at room temperature) in $50 \mathrm{mM} \mathrm{NH} \mathrm{HCO}_{3}$. After digestion, the derivatization reaction was performed again twice to derivatize peptide $\mathrm{N}$-termini. Samples were desalted by using C18 Stage-tips, and resuspended in $0.1 \%$ formic acid for LC-MS analysis.

\section{Quantitative mass spectrometry}

Histone samples were analyzed using nano liquid chromatography-tandem mass spectrometry (nanoLCMS/MS) essentially as described previously [27]. Briefly, nanoLC was configured with a $75 \mu \mathrm{m} \mathrm{ID} \times 17 \mathrm{~cm}$ Reprosil-Pur C18-AQ (3 $\mu \mathrm{m}$; Dr. Maisch GmbH, Germany) nano-column using an EASY-nLC nanoHPLC (Thermo Scientific, Odense, Denmark). Detection was performed by using an Orbitrap XL mass spectrometer (Thermo Scientific, Odense, Denmark). Peak area was extracted from raw files by using our in-house software EpiQuant. The relative abundance of a given PTM was calculated by dividing its intensity by the sum of all modified and unmodified peptides sharing the same sequence. All raw files are available at the Chorus database (https://chorusproject.org).

\section{Determination of variant histone relative abundance}

In order to determine the fraction of canonical histone $\mathrm{H} 2 \mathrm{~A}$, macroH2A, and H2A.Z histones that comprises fH3.1-, fH3.3-, fH2Ac-, and fH2A.Z-containing mononucleosomes, we first quantified four peptides within the canonical histone $\mathrm{H} 2 \mathrm{~A}$ protein, namely the abundances of 4-11 (GKQGGKAR; H2Ac 1 ), 12-17 (AKAKTR; H2Ac 2 ), 21-29 (AGLQFPVGR; $\mathrm{H}_{2} \mathrm{Ac}_{3}$ ), and 82-88 (HLQLAIR; $\mathrm{H}_{2} \mathrm{Ac}_{4}$ ) peptides in each sample, which we refer to as reference peptides. Next, we quantified the abundances of the variant-specific peptides, namely the 36-42 canonical histone H2A peptide (KGNYAER; H2Ac), the 4-14 macroH2A peptide (GGKKKSTKTSR), and the 1-19 H2A.Z peptide (AGGKAGKDSGKAKTKAVSR). We then normalized a

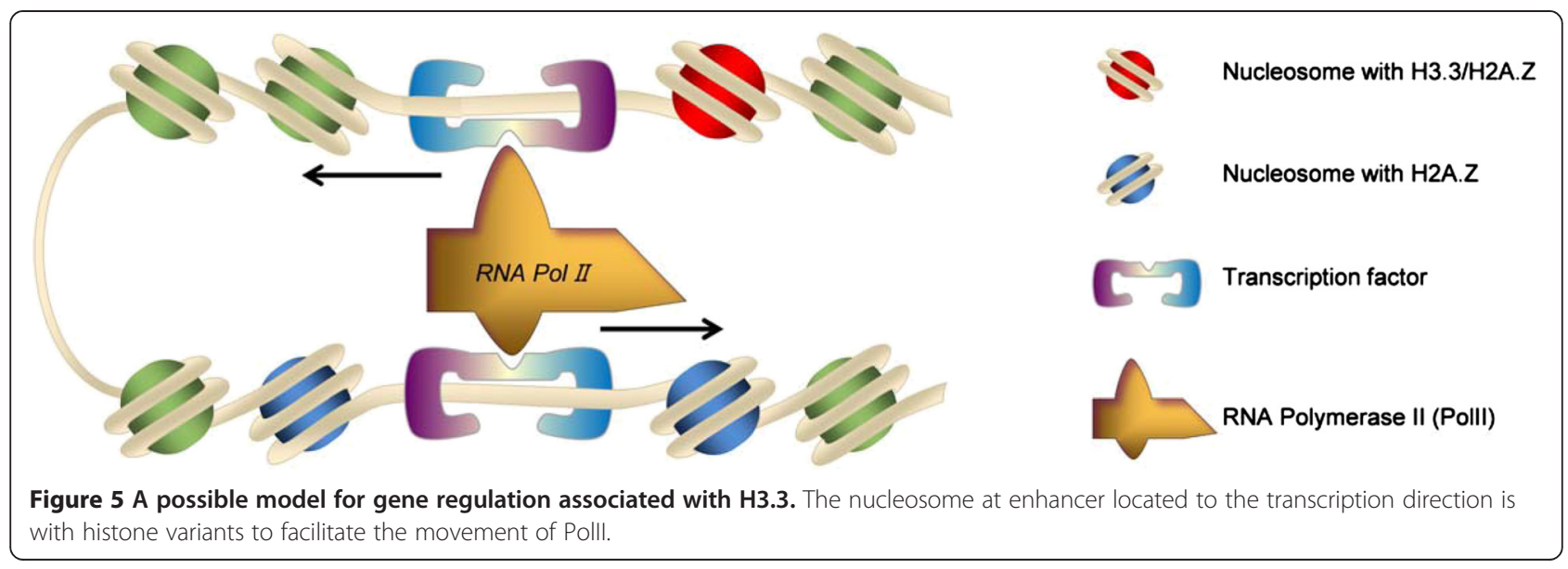


given variant-specific peptide such as the 1-19 H2A.Z peptide over each of the four references to attain four separate ratios, namely a common H2A.Z numerator over four different denominators (that is, $\mathrm{H} 2 \mathrm{~A} . \mathrm{Z} / \mathrm{H} 2 \mathrm{Ac}_{1}$, H2A.Z/H2Ac 2 , H2A.Z/H2Ac,$H 2 A . Z / H 2 A c_{4}$ ). We repeated this normalization for the other variant-specific peptides. To determine the relative ratios of the $\mathrm{H} 3$ variants, we quantified a peptide common to $\mathrm{H} 3.1$ and H3.2 27-40 (KSAPATGGVKKPHR) and compared it directly to the H3.3 peptide 27-40 (KSAPSTGGVKKPHR) that differs by only a single amino acid at residue 31 (alanine in H3.1 and H3.2 and serine in H3.3).

\section{Genomic data processing}

All ChIP-seq tags were aligned to the human genome hg19 using Bowtie [63] with options '-v 2 -m 1 -best -strata, and all of the redundant tags were removed before downstream analysis. All ChIP-seq data were normalized to 10 reads per kilobase per million mapped reads (RPKM) [64]. Besides H3.1 and H3.3, we used ChIP-seq data for macroH2A [32], H2A.Z H3K4me1, and H3K27ac [33]. Additional file 1: Table S1 summarizes all the data we integrated in our study. Besides, we used the ChIPseq data for various transcription factors (TFs) and co-factors from ENCODE database [33] to investigate enriched binding for each cluster. We used the processed peak files deposited in the ENCODE database for this analysis. Hyper-geometric $P$ values were calculated to identify enriched occupancy for TFs and cofactors. We used Refseq annotated genes (hg19) and sort them based on expression level from RNAseq data in HeLa cells [33]. DHSs were identified using Homer [35]. We excluded DHSs at promoter or body of annotated genes in GENCODE v19 [65]. Clustering was performed using the obtained distal DHSs. For clustering analysis, we used MeV V4.8 [66] and applied the $K$-means clustering algorithm to the ChIP-seq data of histone variants (H3.1, H3.3, macroH2A, and H2A.Z) using the Pearson correlation with absolute distance as a metric $(K=10)$. Clusters were rearranged to group the mirroring images. Average profiles were obtained using the Homer package [35].

\section{Data availability}

The ChIP-seq data from this study are available at the NCBI Gene Expression Omnibus (GEO; http://www.ncbi. nlm.nih.gov/geo/) under accession number (GSE64652).

\section{Additional files}

Additional file 1: Table S1. The ChIP-seq data sets included in the study. Previous ChIP-Seq data sets that were used and compared with our results.

Additional file 2: Table S2. Relative abundance of histone modifications from FLAG-IP-purified samples. Quantification normalized histone PTM relative abundances from the mass spectrometry data.
Additional file 3: Figure S1. The averaged profiles of histone variants in association with gene expression. Figure $\mathbf{S} 2$. Histone variants' codes and their shapes. Figure S3. Histone variants and other factors. Figure S4. Polll is enriched to the direction of H3.3. Figure S5. Comparison of eNA levels and Polll levels. Figure S6. Transcription factors and co-factors enriched at each cluster significantly enriched at each cluster. Figure S7. The averaged profiles of H3.3, H2A.Z, and Polll at the TSSs of active genes.

\section{Abbreviations}

CAGE: cap analysis of gene expression; ChIP: chromatin immunoprecipitation; ChIP-MS: ChIP followed by mass spectrometry; ChIP-seq: ChIP followed by next-generation sequencing; DHS: DNasel hypersensitive site: eRNA: enhancer RNA; GEO: gene expression omnibus; GRO-seq: global run-on sequencing; IncRNA: long non-coding RNA; mESC: mouse embryonic stem cell; NCP: nucleosome core particle; NFR: nucleosome free regions; PTM: post-translational modification; Polll: RNA polymerase II; RPKM: reads per kilobase per million mapped reads; TF: transcription factor;

TSS: transcriptional start site; TTS: transcription termination site.

\section{Competing interests}

The authors declare that they have no competing interests.

\section{Authors' contributions}

KJW and BAG conceived the project. KJW and IC performed the bioinformatics analyses. GL, BMZ, and BAG performed the mass spectrometry experiment. MGC validated our results using PCR. SS contributed to the mass spectrometry data analysis and also in the manuscript preparation. KJW, GL, and BAG wrote the manuscript. All authors read and approved the final manuscript.

\section{Acknowledgements}

BAG acknowledges funding from an NIH grant R01GM110174 and an NSF Faculty CAREER award. KJW acknowledges funding from an NIH/NIDDK grant R21DK098769-01.

\section{Author details}

'The Institute for Diabetes, Obesity, and Metabolism, Philadelphia, PA 19104 USA. ${ }^{2}$ Department of Genetics, Perelman School of Medicine, University of Pennsylvania, Philadelphia, PA 19104, USA. ${ }^{3}$ Department of Molecular Biology, Princeton University, Princeton, NJ 08544, USA. ${ }^{4}$ Epigenetics Program, Department of Biochemistry and Biophysics, Perelman School of Medicine, University of Pennsylvania, Philadelphia, PA 19104, USA.

${ }^{5}$ Department of Agricultural Biotechnology, National Academy of Agricultural Science, 370 Nongsaengmyeong-ro, Wansan-gu, Jeonju-si, Jeollabuk-do 560-500, South Korea.

Received: 13 February 2015 Accepted: 19 March 2015

Published online: 10 April 2015

\section{References}

1. Li B, Carey M, Workman JL. The role of chromatin during transcription. Cell. 2007;128:707-19.

2. Luger K. Structure and dynamic behavior of nucleosomes. Curr Opin Genet Dev. 2003:13:127-35.

3. Xu D, Bai J, Duan Q, Costa M, Dai W. Covalent modifications of histones during mitosis and meiosis. Cell Cycle. 2009;8:3688-94.

4. Bannister AJ, Kouzarides T. Regulation of chromatin by histone modifications. Cell Res. 2011;21:381-95.

5. Alabert C, Groth A. Chromatin replication and epigenome maintenance. Nat Rev Mol Cell Biol. 2012;13:153-67.

6. Portela A, Esteller M. Epigenetic modifications and human disease. Nat Biotechnol. 2010;28:1057-68.

7. Chi P, Allis CD, Wang GG. Covalent histone modifications-miswritten, misinterpreted and mis-erased in human cancers. Nat Rev Cancer. 2010;10:457-69.

8. Kamakaka RT, Biggins S. Histone variants: deviants? Genes Dev. 2005;19:295-310.

9. Weber CM, Henikoff S. Histone variants: dynamic punctuation in transcription. Genes Dev. 2014;28:672-82. 
10. Adam M, Robert F, Larochelle M, Gaudreau L. H2A.Z is required for global chromatin integrity and for recruitment of RNA polymerase II under specific conditions. Mol Cell Biol. 2001;21:6270-9.

11. Barski A, Cuddapah S, Cui K, Roh TY, Schones DE, Wang Z, et al. Highresolution profiling of histone methylations in the human genome. Cell. 2007;129:823-37.

12. Guillemette B, Bataille AR, Gevry N, Adam M, Blanchette M, Robert F, et al. Variant histone H2A.Z is globally localized to the promoters of inactive yeast genes and regulates nucleosome positioning. PLoS Biol. 2005;3:e384.

13. Santisteban MS, Kalashnikova T, Smith MM. Histone H2A.Z regulates transcription and is partially redundant with nucleosome remodeling complexes. Cell. 2000;103:411-22.

14. Zhang H, Roberts DN, Cairns BR. Genome-wide dynamics of Htz1, a histone $\mathrm{H} 2 \mathrm{~A}$ variant that poises repressed/basal promoters for activation through histone loss. Cell. 2005;123:219-31.

15. Raisner RM, Hartley PD, Meneghini MD, Bao MZ, Liu CL, Schreiber SL, et al. Histone variant H2A.Z marks the $5^{\prime}$ ends of both active and inactive genes in euchromatin. Cell. 2005;123:233-48.

16. Ku M, Jaffe JD, Koche RP, Rheinbay E, Endoh M, Koseki H, et al. H2A.Z landscapes and dual modifications in pluripotent and multipotent stem cells underlie complex genome regulatory functions. Genome Biol. 2012;13:R85.

17. Hu G, Cui K, Northrup D, Liu C, Wang C, Tang Q, et al. H2A.Z facilitates access of active and repressive complexes to chromatin in embryonic stem cell self-renewal and differentiation. Cell Stem Cell. 2013;12:180-92.

18. Mito Y, Henikoff JG, Henikoff S. Genome-scale profiling of histone H3.3 replacement patterns. Nat Genet. 2005;37:1090-7.

19. Mito $Y$, Henikoff JG, Henikoff S. Histone replacement marks the boundaries of cis-regulatory domains. Science. 2007;315:1408-11.

20. Ahmad K, Henikoff S. The histone variant H3.3 marks active chromatin by replication-independent nucleosome assembly. Mol Cell. 2002;9:1191-200.

21. Goldberg AD, Banaszynski LA, Noh KM, Lewis PW, Elsaesser SJ, Stadler S, et al. Distinct factors control histone variant H3.3 localization at specific genomic regions. Cell. 2010;140:678-91.

22. Ray-Gallet D, Woolfe A, Vassias I, Pellentz C, Lacoste N, Puri A, et al. Dynamics of histone $\mathrm{H} 3$ deposition in vivo reveal a nucleosome gap-filling mechanism for H3.3 to maintain chromatin integrity. Mol Cell. 2011:44:928-41.

23. Jin C, Zang C, Wei G, Cui K, Peng W, Zhao K, et al. H3.3/H2A.Z double variant-containing nucleosomes mark 'nucleosome-free regions' of active promoters and other regulatory regions. Nat Genet. 2009;41:941-5.

24. Changolkar LN, Pehrson JR. macroH2A1 histone variants are depleted on active genes but concentrated on the inactive $\mathrm{X}$ chromosome. Mol Cell Biol. 2006:26:4410-20.

25. Costanzi $\mathrm{C}$, Pehrson JR. Histone macroH2A1 is concentrated in the inactive X chromosome of female mammals. Nature. 1998;393:599-601.

26. Gamble MJ, Frizzell KM, Yang C, Krishnakumar R, Kraus WL. The histone variant macroH2A1 marks repressed autosomal chromatin, but protects a subset of its target genes from silencing. Genes Dev. 2010;24:21-32.

27. LeRoy G, Chepelev I, DiMaggio PA, Blanco MA, Zee BM, Zhao K, et al. Proteogenomic characterization and mapping of nucleosomes decoded by Brd and HP1 proteins. Genome Biol. 2012;13:R68.

28. Wang Z, Zang C, Rosenfeld JA, Schones DE, Barski A, Cuddapah S, et al. Combinatorial patterns of histone acetylations and methylations in the human genome. Nat Genet. 2008;40:897-903.

29. LeRoy G, Rickards B, Flint SJ. The double bromodomain proteins Brd2 and Brd3 couple histone acetylation to transcription. Mol Cell. 2008:30:51-60.

30. Tolstorukov MY, Kharchenko PV, Goldman JA, Kingston RE, Park PJ. Comparative analysis of H2A.Z nucleosome organization in the human and yeast genomes. Genome Res. 2009;19:967-77.

31. Kaplan N, Moore IK, Fondufe-Mittendorf Y, Gossett AJ, Tillo D, Field Y, et al The DNA-encoded nucleosome organization of a eukaryotic genome. Nature. 2009;458:362-6.

32. Tolstorukov MY, Goldman JA, Gilbert C, Ogryzko V, Kingston RE, Park PJ. Histone variant $\mathrm{H} 2 \mathrm{~A} . \mathrm{Bbd}$ is associated with active transcription and mRNA processing in human cells. Mol Cell. 2012;47:596-607.

33. The ENCODE Project Consortium. An integrated encyclopedia of DNA elements in the human genome. Nature. 2012:489:57-74

34. Song L, Crawford GE. DNase-seq: a high-resolution technique for mapping active gene regulatory elements across the genome from mammalian cells. Cold Spring Harb Protoc. 2010. doi:10.1101/pdb.prot5384.

35. Heinz S, Benner C, Spann N, Bertolino E, Lin YC, Laslo P, et al. Simple combinations of lineage-determining transcription factors prime cis- regulatory elements required for macrophage and B cell identities. Mol Cell. 2010:38:576-89.

36. Creyghton MP, Cheng AW, Welstead GG, Kooistra T, Carey BW, Steine EJ, et al. Histone H3K27ac separates active from poised enhancers and predicts developmental state. Proc Natl Acad Sci U S A. 2010;107:21931-6.

37. Heintzman ND, Stuart RK, Hon G, Fu Y, Ching CW, Hawkins RD, et al. Distinct and predictive chromatin signatures of transcriptional promoters and enhancers in the human genome. Nat Genet. 2007;39:311-8.

38. May D, Blow MJ, Kaplan T, McCulley DJ, Jensen BC, Akiyama JA, et al. Largescale discovery of enhancers from human heart tissue. Nat Genet. 2011;44:89-93.

39. Andersson R, Refsing Andersen P, Valen E, Core LJ, Bornholdt J, Boyd M, et al. Nuclear stability and transcriptional directionality separate functionally distinct RNA species. Nat Commun. 2014;5:5336.

40. Wang D, Garcia-Bassets I, Benner C, Li W, Su X, Zhou Y, et al. Reprogramming transcription by distinct classes of enhancers functionally defined by eRNA. Nature. 2011:474:390-4.

41. Kim TK, Hemberg M, Gray JM, Costa AM, Bear DM, Wu J, et al. Widespread transcription at neuronal activity-regulated enhancers. Nature. 2010;465:182-7.

42. Jin C, Felsenfeld G. Nucleosome stability mediated by histone variants H3.3 and H2A.Z. Genes Dev. 2007:21:1519-29.

43. Phillips JE, Corces VG. CTCF: master weaver of the genome. Cell. 2009:137:1194-211.

44. Won KJ, Chepelev I, Ren B, Wang W. Prediction of regulatory elements in mammalian genomes using chromatin signatures. BMC Bioinformatics. 2008;9:547.

45. Won KJ, Ren B, Wang W. Genome-wide prediction of transcription factor binding sites using an integrated model. Genome Biol. 2010;11:R7.

46. Andersson R, Gebhard C, Miguel-Escalada I, Hoof I, Bornholdt J, Boyd M, et al. An atlas of active enhancers across human cell types and tissues. Nature. 2014;507:455-61.

47. Banerii J, Rusconi S, Schaffner W. Expression of a beta-globin gene is enhanced by remote SV40 DNA sequences. Cell. 1981;27:299-308.

48. Kundaje A, Kyriazopoulou-Panagiotopoulou S, Libbrecht M, Smith CL, Raha D, Winters $\mathrm{EE}$, et al. Ubiquitous heterogeneity and asymmetry of the chromatin environment at regulatory elements. Genome Res. 2012;22:1735-47.

49. Lai WK, Buck MJ. ArchAlign: coordinate-free chromatin alignment reveals novel architectures. Genome Biol. 2010;11:R126.

50. Nielsen FG, Markus KG, Friborg RM, Favrholdt LM, Stunnenberg HG, Huynen M. CATCHprofiles: clustering and alignment tool for ChIP profiles. PLoS One. 2012; 7, e28272.

51. Li W, Notani D, Ma Q, Tanasa B, Nunez E, Chen AY, et al. Functional roles of enhancer RNAs for oestrogen-dependent transcriptional activation. Nature. 2013;498:516-20

52. Min IM, Waterfall JJ, Core LJ, Munroe RJ, Schimenti J, Lis JT. Regulating RNA polymerase pausing and transcription elongation in embryonic stem cells. Genes Dev. 2011:25:742-54.

53. Core $L$, Waterfall JJ, Lis JT. Nascent RNA sequencing reveals widespread pausing and divergent initiation at human promoters. Science. 2008;322:1845-8.

54. Lam MT, Cho H, Lesch HP, Gosselin D, Heinz S, Tanaka-Oishi Y, et al. Rev-Erbs repress macrophage gene expression by inhibiting enhancer-directed transcription. Nature. 2013;498:511-5.

55. Bargaje R, Alam MP, Patowary A, Sarkar M, Ali T, Gupta S, et al. Proximity of H2A.Z containing nucleosome to the transcription start site influences gene expression levels in the mammalian liver and brain. Nucleic Acids Res. 2012:40:8965-78

56. Mavrich TN, Jiang C, loshikhes IP, Li X, Venters BJ, Zanton SJ, et al. Nucleosome organization in the Drosophila genome. Nature. 2008;453:358-62.

57. Zilberman D, Coleman-Derr D, Ballinger T, Henikoff S. Histone H2A.Z and DNA methylation are mutually antagonistic chromatin marks. Nature. 2008:456:125-9.

58. Xu Z, Wei W, Gagneur J, Perocchi F, Clauder-Munster S, Camblong J, et al. Bidirectional promoters generate pervasive transcription in yeast. Nature. 2009;457:1033-7

59. Weber CM, Ramachandran S, Henikoff S. Nucleosomes are context-specific H2A.Z-modulated barriers to RNA polymerase. Mol Cell. 2014;53:819-30.

60. Blackwood EM, Kadonaga JT. Going the distance: a current view of enhancer action. Science. 1998;281:60-3.

61. Krivega I, Dean A. Enhancer and promoter interactions-long distance calls. Curr Opin Genet Dev. 2012;22:79-85. 
62. Zhang Y, Wong CH, Birnbaum RY, Li G, Favaro R, Ngan CY, et al. Chromatin connectivity maps reveal dynamic promoter-enhancer long-range associations. Nature. 2013;504:306-10.

63. Langmead B, Trapnell C, Pop M, Salzberg SL. Ultrafast and memory-efficient alignment of short DNA sequences to the human genome. Genome Biol. 2009;10:R25.

64. Mortazavi A, Williams BA, McCue K, Schaeffer L, Wold B. Mapping and quantifying mammalian transcriptomes by RNA-Seq. Nat Methods. 2008;:5:621-8.

65. Harrow J, Frankish A, Gonzalez JM, Tapanari E, Diekhans M, Kokocinski F, et al. GENCODE: the reference human genome annotation for The ENCODE Project. Genome Res. 2012;22:1760-74.

66. Saeed Al, Bhagabati NK, Braisted JC, Liang W, Sharov V, Howe EA, et al. TM4 microarray software suite. Methods Enzymol. 2006:411:134-93.

\section{Submit your next manuscript to BioMed Central and take full advantage of:}

- Convenient online submission

- Thorough peer review

- No space constraints or color figure charges

- Immediate publication on acceptance

- Inclusion in PubMed, CAS, Scopus and Google Scholar

- Research which is freely available for redistribution 